\title{
Computer-aided design system aids mammography screening
}

\author{
San-Kan Lee, Chien-Shun Lo, Jia-Pei Huang, Giu-Cheng Hsu, \\ Mann-Li Chang, and Pau-Choo (Julia) Chung
}

A computerized tool gives radiologists a second opinion for diagnosing breast cancer.

The drive for early breast cancer detection has significantly increased the number of mammograms radiologists must read. An effective computer-aided design (CAD) system for screening can help relieve that workload and improve diagnosis. Several US Food and Drug Administration-approved CAD systems are currently being used with digital mammography. They can provide detection results, but not classification according to the five categories of the American College of Radiology's Breast Imaging Reporting and Data System (BIRADS). This is primarily because sophisticated characteristics in mammograms are challenging for a CAD system to classify effectively.

For example, microcalcifications (MCCs) and masses exhibit completely different characteristics. MCCs are generally made by foreign substances, not breast tissues. Their presence can be detected by intensity and contrast, and their classification is based on their size and shape. However, masses are considered part of breast tissues. They can only be detected by their texture features, which can be used for classification. Thus, different design techniques are required for each abnormality. Our CAD pilot system is designed to detect and classify MCCs and masses in parallel, using two sets of different techniques. ${ }^{1}$

Our system uses five modules: preprocessing, MCCs/masses finding, MCCs/masses detection, MCCs/masses classification, and audit/reporting. A step-by-step implementation of each is shown in Figure 1.2,3 The pre-processing module digitizes a mammogram into an 8-bit image with $2048 \times 2048$ pixels, as shown in Figure 1(a). It extracts the breast region from the mammogram, enhances that image, and stores the processed and the original unprocessed mammograms in a database for future reference. Since only clustered MCCs or masses provide useful diagnostic information about malignancy, the second module locates suspicious groupings, then segments them from the background image as regions of interest (ROIs) to be

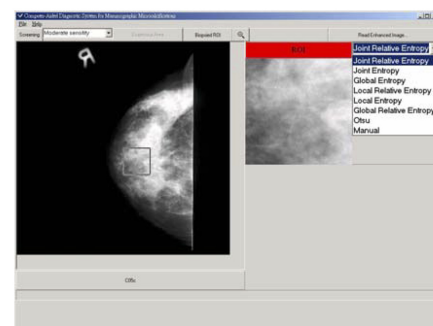

(a) pre-processing module

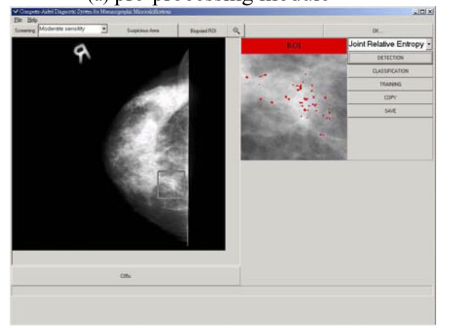

(c) MCCs/masses detection module

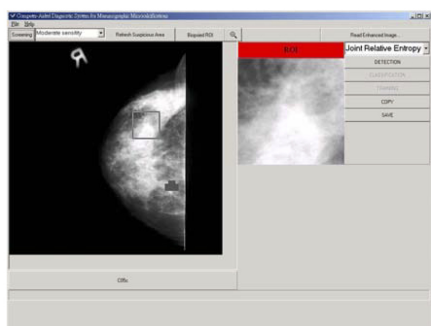

(b) MCCs/masses finding module

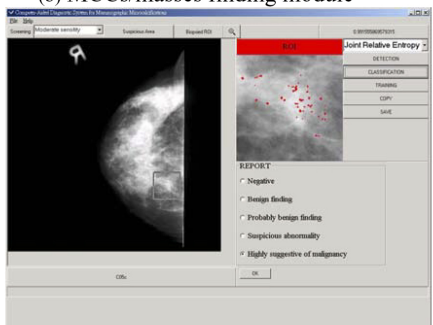

(d) MCCs/masses classification module

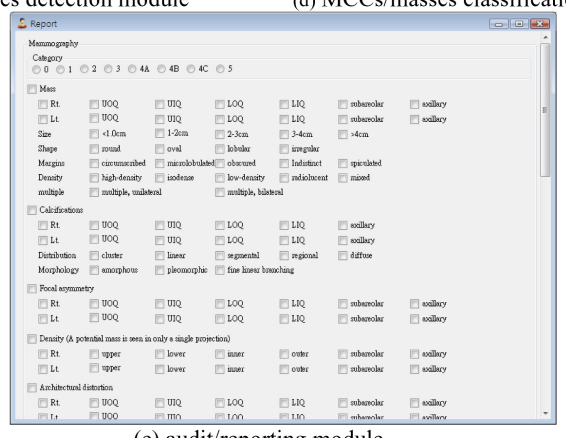

(e) audit/reporting module

Figure 1. The five modules in a prototype computer-aided design (CAD) mammography screening system.

used for further detection. A suspicious shaded area is shown in Figure 1(b). As soon as ROIs are identified, they are fed into the detection module for processing, as seen in Figure 1(c). This module is a real-time processing system that uses two different window sizes to extract MCCs/masses. It begins with a large $64 \times 64$ pixel window to quickly screen mammograms to find large, suspicious areas. This is followed by a smaller 

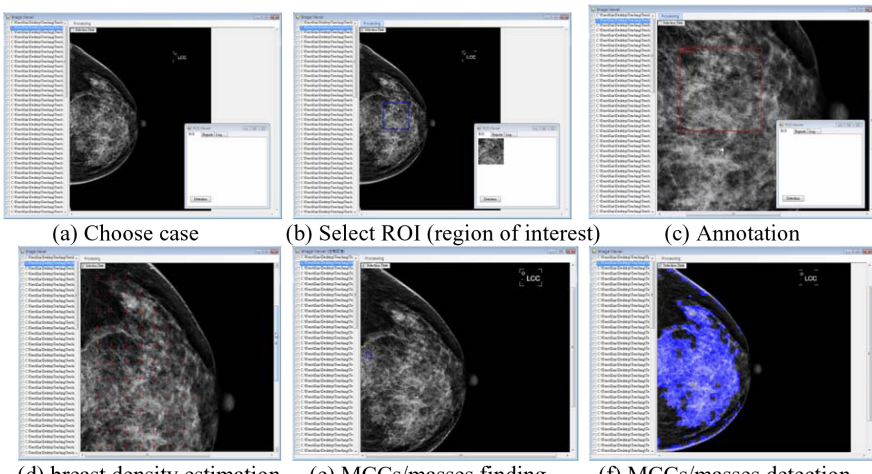

(b) Select ROI (region of interest)

(c) Annotation
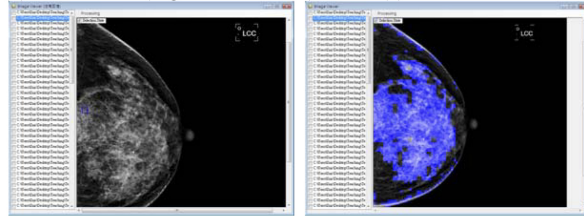

$\begin{array}{ll}\text { (d) breast density estimation } & \text { (e) MCCs/masses finding }\end{array}$

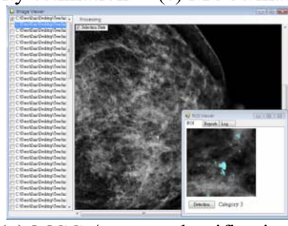

(g) MCCs/masses classification

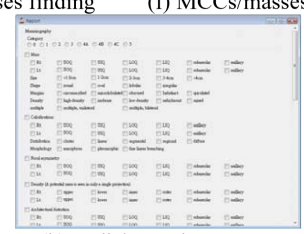

(h) audit/reporting system
Figure 2. A step-by-step CAD mammography screening system including an estimation of breast density.

$8 \times 8$ pixel window to extract small and tiny MCCs/masses. The module shown in Figure 1(d) classifies the segmented clustered MCCs/masses into one of five results: 'negative' (no further operation), 'benign' (MCCs/masses found to be negative), 'probably benign' (short interval follow-up suggested), 'suspicious abnormality' (biopsy should be considered), and 'highly suggestive of malignancy' (appropriate action should be taken). Finally, the audit/reporting module allows doctors to edit and write their reports as shown in Figure 1(e).

Our system moves one step further toward improving current CAD mammography screening by classifying MCCs/masses according to the BIRADS guideline. Nevertheless, it is still a prototype, and much work remains. Specifically, we will further study BIRADS analysis. For example, density is one feature used to evaluate breast cancer. However, it is difficult for doctors to quantify breast density. We are proposing a quantification tool for breast density in our CAD system (see Figure 2), and we also will study other BIRADS density evaluation tools.

\section{Author Information}

\section{San-Kan Lee}

Department of Radiology

Taichung Veterans General Hospital

Taichung, Taiwan
San-Kan Lee is a professor of Radiology at National Defense Medical Center and Chung Shan Medical University, and a Deputy Superintendent at Taichung Veterans General Hospital. His research includes computed-aided diagnosis in mammograms, screening of breast cancer, and picture archiving and communication systems. He holds one US and one Taiwan patent.

\section{Chien-Shun Lo \\ Department of Multimedia Design \\ National Formosa University \\ Yunlin County, Taiwan}

Chien-Shun Lo, assistant professor in the Department of Multimedia Design, received his BS and MS in information engineering and computer science from Feng-Chia University in 1992 and 1994, respectively, and his $\mathrm{PhD}$ in electrical engineering from National Cheng-Kung University in 2000. His current research includes computer-aided diagnostic system design, image processing, video processing, and streaming technology.

\section{Jia-Pei Huang}

School of Computer Science and Information Engineering National Cheng Kung University (NCKU)

Tainan, Taiwan

Jia-Pei Huang received his MS in computer science and information engineering from National Chiayi University, Taiwan, in 2005. He is currently pursuing his PhD in the School of Computer Science and Information Engineering from NCKU. His research interests include computer-aided diagnosis in mammograms, pattern recognition, neural networks, computer vision, and their applications.

\section{Giu-Cheng Hsu \\ Department of Diagnostic Radiology \\ Tri-Service General Hospital (TSGH) \\ Taipei, Taiwan}

Giu-Cheng Hsu served in the Department of Diagnostic Radiology at TSGH from 1985 to 1996. He was director of the Department of Diagnostic Radiology at Taiwan Adventist Hospital from 1996 to 2001. He returned to TSGH in 2001 as a director, and established the Women's Health Center. 


\section{Mann-Li Chang}

Information System Division

Bureau of National Health Insurance (BNHI)

Taipei, Taiwan

Mann-Li Chang received her BS and MS from Chinese Culture University in Taiwan, her Management Information Systems Program degree from Bowie State University in the US, and her PhD from National Normal University in Taiwan. She is currently working for the BNHI's Information System Division and is a part-time faculty member at National Open University Taipei and Kinmen Center. Her research includes information systems, sciences and management, and remote sensing image processing.

\section{Pau-Choo (Julia) Chung}

Institute of Computer and Communication Engineering

National Cheng Kung University (NCKU)

Tainan, Taiwan

Pau-Choo (Julia) Chung is the director of the Institute of Computer and Communication Engineering, NCKU. She received her BS and MS degrees in electrical engineering from NCKU and her $\mathrm{PhD}$ in electrical engineering from Texas Tech University in the US. She has been a full professor of the Department of Electrical Engineering at NCKU since 1996. She is a member of Phi Tau Phi honor society and an IEEE fellow.

\section{References}

1. Y. W. Chang, Y. C. Chang, C.-I. Chang, G.-C. Hsu, H.-H. Hsu, S.-M. Guo, S.-C. Yang, P.-C. Chung, and S.-K. Lee, Chapter 3: Computer-aided diagnosis for breast cancer detection by mammography, S.G. Pandalai ed., Chapter 3, Recent research developments in biomedical engineering 2, pp. 53-92, Trivandrum, Kerala: Research Signpost, India, 2005.

2. S.-K. Lee, C.-S. Lo, C.-M. Wang, P.-C. Chung, C.-I Chang, C.-W. Yang, and P.-C. Hsu, A computer-aided design mammography screening system for detection and classification of microcalcifications, J. Medical Informatics 60 (1), pp. 29-57, October 2000 .

3. S. K. Lee, P.-C. Chung, C.-I Chang, C.-S. Lo, T. Lee, G.-C. Hsu, and C.-W. Yang, Classification of clustered microcalcifications using a shape cognitron, Neural Networks 16, pp. 121-132, January 2003. 